\title{
The Construction of Off-line Map Based on OpenStreetMap and Leaflet
}

\author{
Ludi Wang ${ }^{1, a}$, Renjie $\mathrm{Pi}^{1, \mathrm{~b}}$, Xiaoguang Zhou ${ }^{1, \mathrm{c}}$ and Huiling Zhou ${ }^{1, \mathrm{~d}}$ \\ ${ }^{1}$ School of Automation, BUPT, Beijing 100876, China; \\ a15117928901@163.com, birenjie@gmail.com, 'zxg@bupt.edu.cn, dhuiling@bupt.edu.cn
}

Keywords: OSM (OpenStreetMap) Leaflet off-line map

\begin{abstract}
OSM (OpenStreetMap) is a collaborative project to create a free editable map of the world and Leaflet is the leading open-source JavaScript library for mobile-friendly interactive maps. Based on OSM (OpenStreetMap) and Leaflet, a complete off-line map is constructed in this paper and applied to the actual emergency logistics project, which reduces the dependence of the open source map on the network and improves the security of the system.
\end{abstract}

\section{Introduction}

With the continuous development of intelligent transportation, the role of electronic map in people's daily life is becoming more and more obvious. Electronic map is divided into two types, one is online map application, which need to connect to the corresponding map server and receive relevant tile data; the second one is the offline map application, which need to obtain the required data through offline data. Because the latter can get the map data quickly without access to the network, so it is very important to construct the off line map.

\section{The Introduction of OSM}

At present, China's users are highly praised by Baidu maps, although it has improved, but because it does not provide geographic data outside of China, and is difficult to merge with other foreign open source plugin. So it is difficult for the development of the follow-up development of developers. Compared to the above geographic data, the biggest characteristic and advantage of OSM lies in its openness. The complete opening of the atomic data and the update rate of the data can help the researchers and application developers to resolve some problem and also provide valuable resources for the development of work. Therefore, the research of software development based on OSM data has important practical significance for modern city residents' daily life.

\section{Map data download}

When using the OSM map data to build an offline map, we need to download the basic geographic data from the OSM server, methods are as follows:

Directly download through the official website: OSM official website provides the source data download service, the advantages of this method are convenient and quick, but the disadvantage is that it doesn't support the image or database format file download.

Use open source software for download: Open source software such as Maperitive can download the OSM map data and can convert the data into a PNG format image file or .Mbtiles format SQLITE database file.

Key technical analysis of OSM

(1) Map slicing implementation (Tiles) technology

Electronic map is presented in the form of a large electronic map to cut into a number of $256^{*} 256$ pixels set the size of the map, so that it can be flexible to zoom in operation, because as long as you want to query or search the relevant small square in the region can be loaded.

(2) WebServer Technology

Based on WebServer OSM technology can be used to publish their own map server. 


\section{Leaflet API}

Leaflet is the leading open-source JavaScript library for mobile-friendly interactive maps. Weighing just about $33 \mathrm{~KB}$ of JS, it has all the mapping features most developers ever need.

Leaflet is designed with simplicity, performance and usability in mind. It works efficiently across all major desktop and mobile platforms, can be extended with lots of plugins, has a beautiful, easy to use and well-documented API and a simple, readable source code that is a joy to contribute to.

The each interface of Leaflet API is shown in the following diagram:
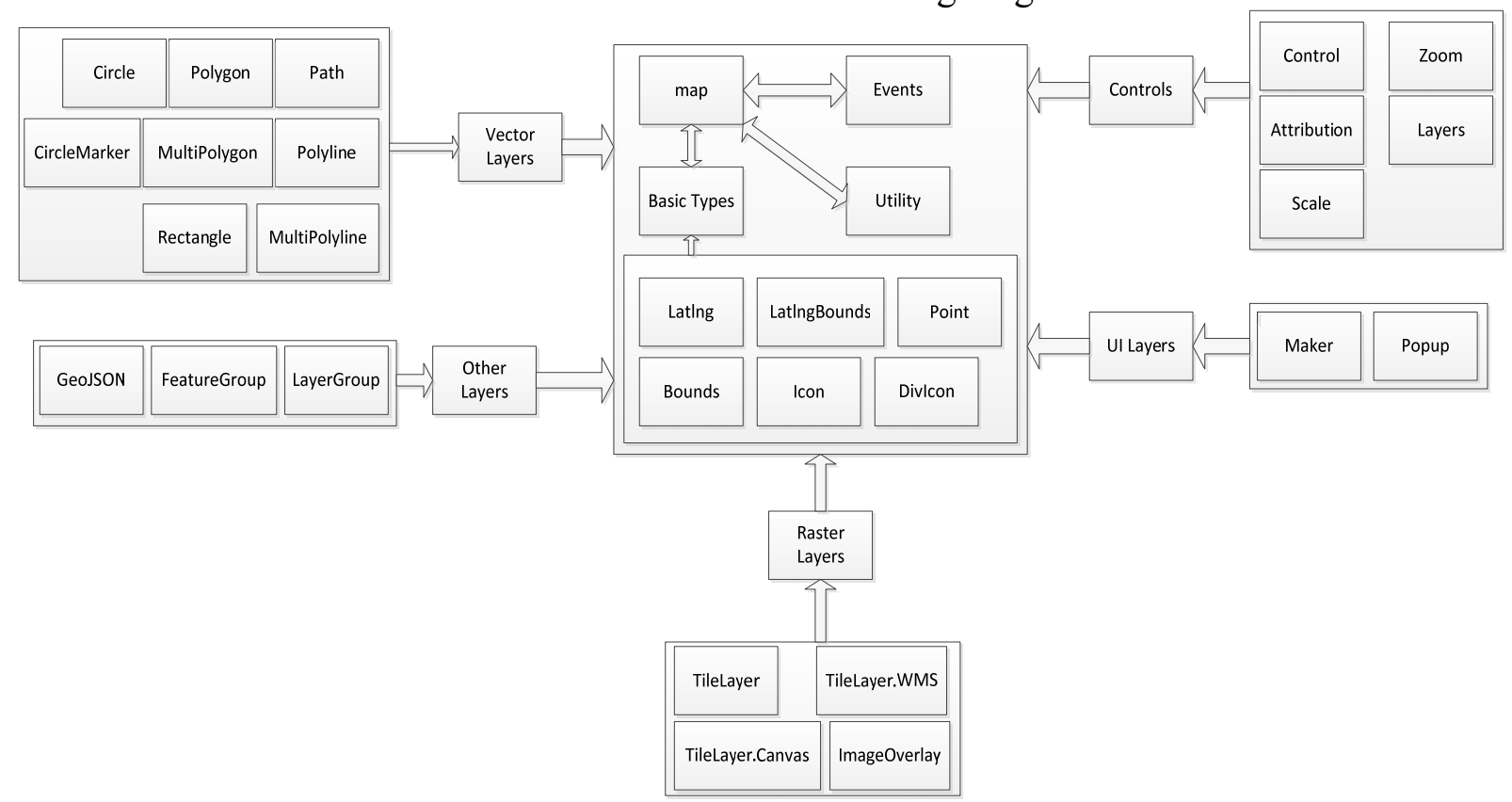

Fig. 1 The Interface of Leaflet API

\section{Overall design of offline map}

The design objective of the offline electronic map based on PC terminal is: (1Fast offline access to map tile data (Tiles) or database tile data (mbtiles); (2) Able to perform other operations on an offline map, such as positioning, navigation, etc.

\section{Overall Architecture}

The main design idea of the application architecture of the offline map which is based on PC is to read local .Mbtiles format files by PHP method, and display files in tiles format on a web page with the JS offered by Leaflet. PC terminal offline map application architecture is shown in Figure 2: 


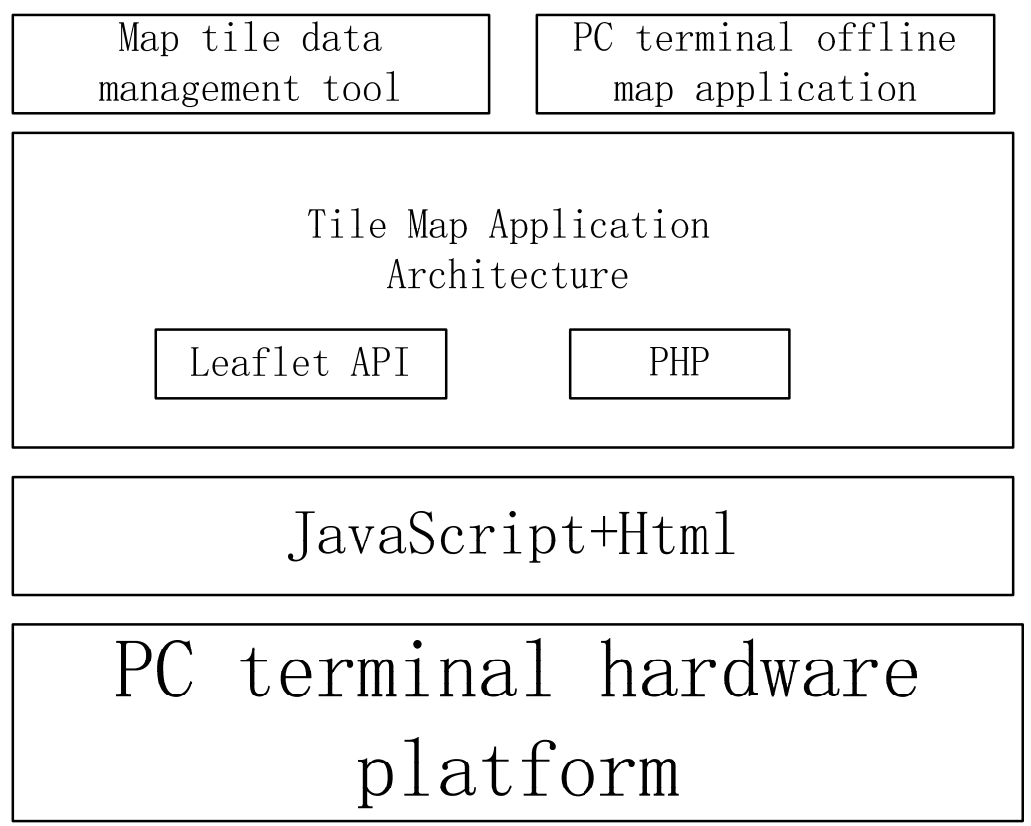

Fig. 2 PC terminal offline map application architecture

In this design, Leaflet API provide the methods to access the tile data, XYZ parameters are calculated by TileLayer function and call the PHP file. The PHP file calls the offline map tile data of the machine by calculating the XYZ parameter. The source code in PHP is as follows:

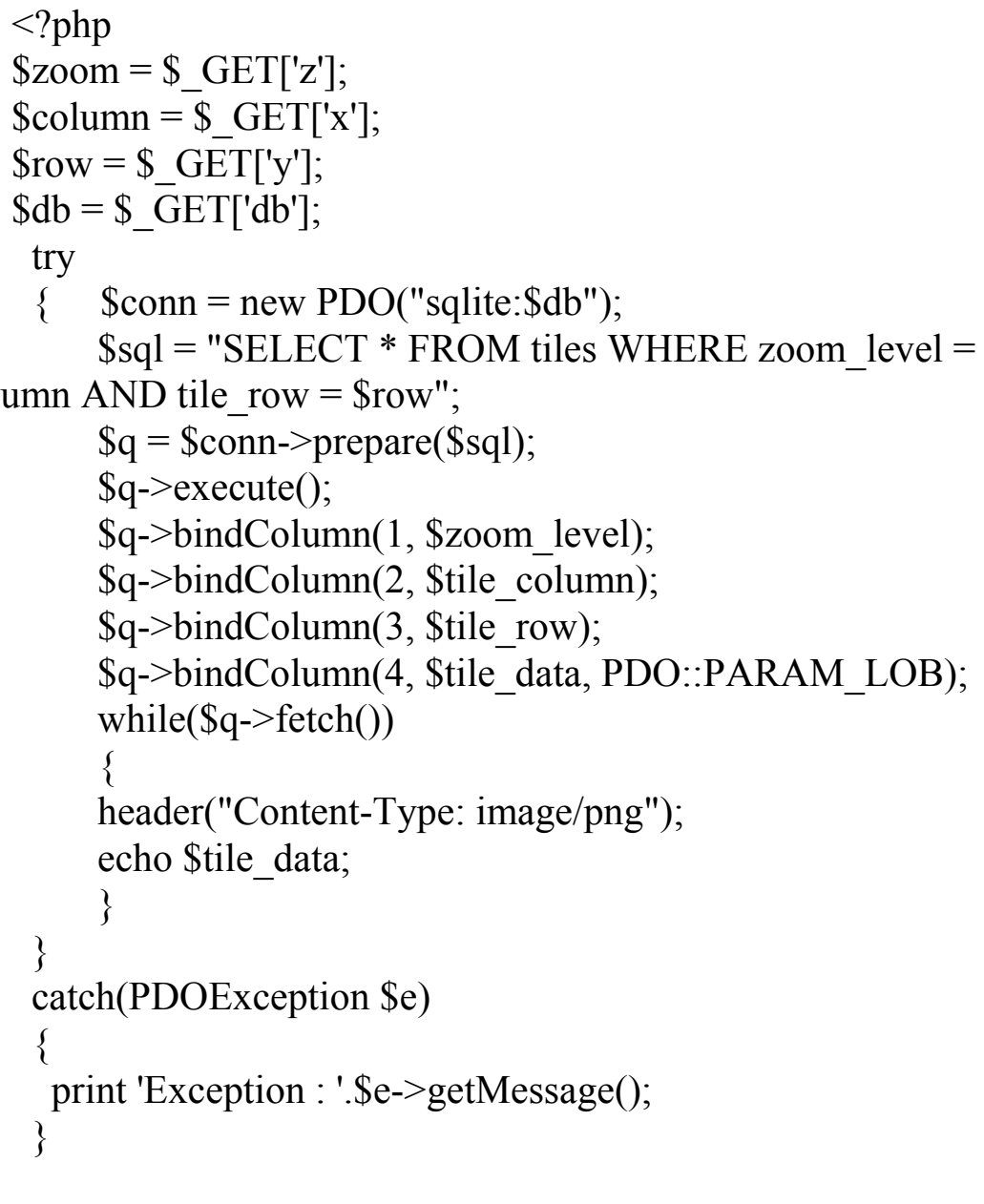

\section{Structure Design}

This structure of the design is as shown in Figure 3: 


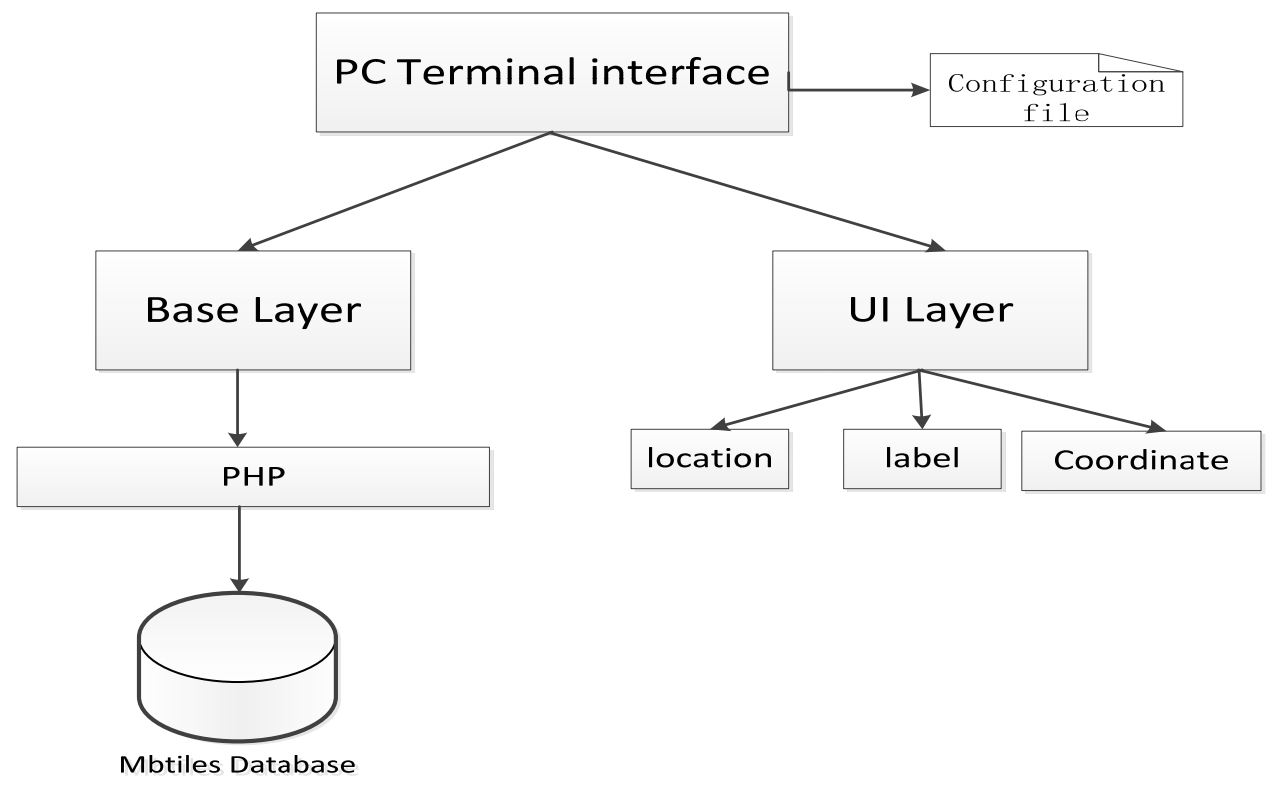

Fig. 3 Structure of the design

The UI interface is initialized by the JS file at the beginning of the web page, including calling tile layer interface, adding UI layer and defining the other interface. According to the zoom level and position center coordinates which are defined by the web page, the XYZ parameters of the corresponding tiles can be calculated with the OSM core logic rule. Finally, the tiles which meet those rules will be displayed on the web page.

After that, we can add layer tools on the map, including Marker, Popup, etc. The main role of these tools is to assist the operator to better use the map file, its function mainly includes the tag position, access coordinates and positioning navigation and so on. System interface is shown in the following figure:

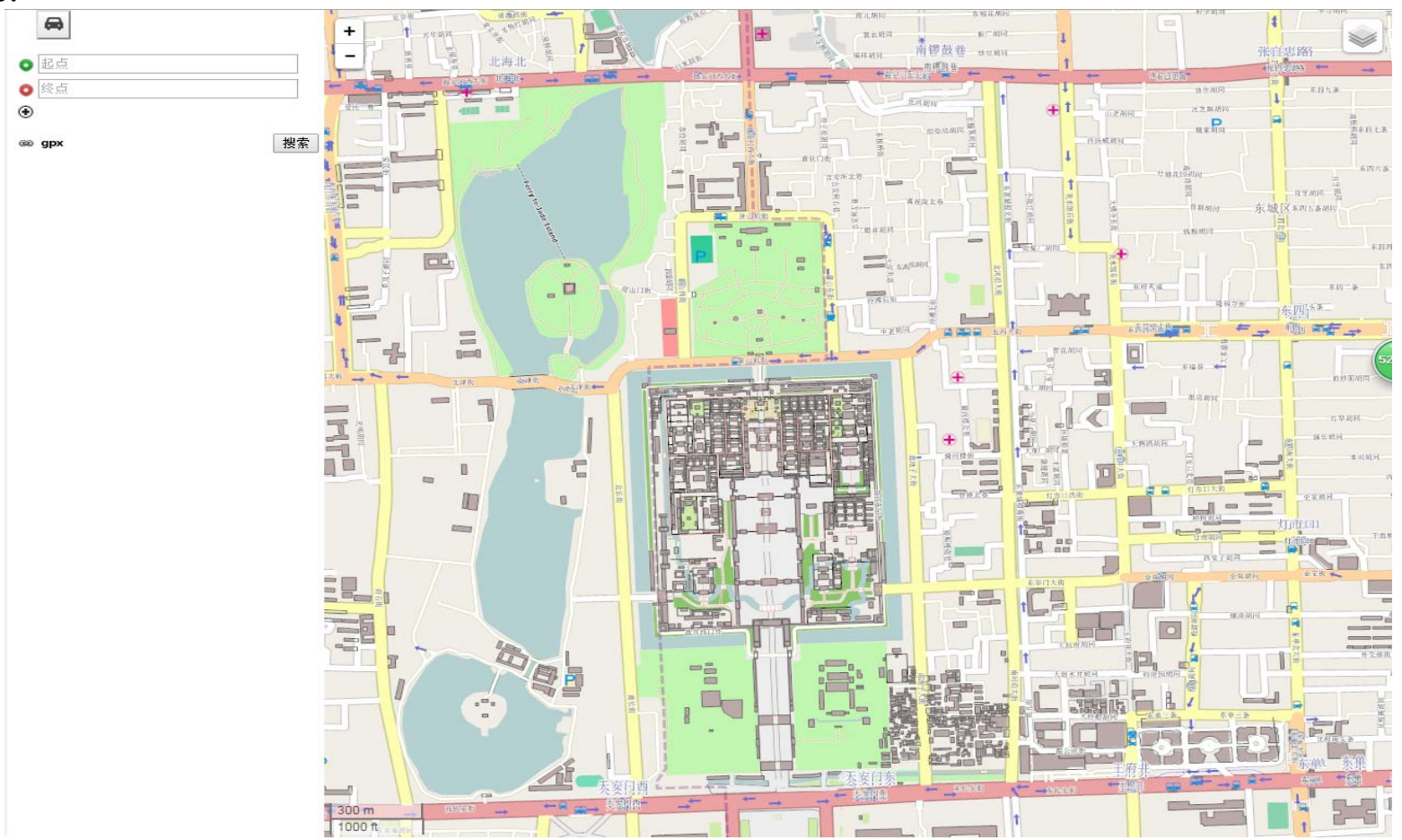

Fig. 4 System interface

\section{Summary}

In view of the present situation of the offline electronic map, this paper designs an offline map system based on the OSM data and Leaflet API, and realizes the access and display of the data. This paper has been applied to the actual offline caching of electronic map items and reflects the following 
advantages: (1)The system can quickly access the electronic map of the offline cache, support the electronic map browsing, navigation and other common functions; (2)Can call the database form of map data, compared with the scattered massive tile file, improve disk utilization and reduce the time to deploy data; (3)Make full use of the Sqlite embedded database, improve the access performance of map tile data. The design further development direction is to combine with the Android client for more practical use.

\section{Acknowledgments}

At this point, this thesis is to come to an end. Here, thanks for the help of the project group, the visual logistics 201313009-08 and all of the colleague in team. There are still many deficiencies in this system, we will continue to develop it in the future.

\section{References}

[1] Pingping Zhou, Wei Huang, Jie Jang, VALIDATION ANALYSIS OF OPENSTREETMAP DATA IN SOME AREAS OF CHINA 2014

[2] Jinming Wei, Fei Shao, Weizheng Zhong, Map service method based on TianDiTu map, Surveying and mapping, 2014(S1): 265-268.DOI:10.3474/j.cnki.11-2246.2014.0711

[3] Kevin Tatroe, Peter MacIntyre, Rasmus Lerdorf, Programming PHP, Electronic Industry Press, China, 2015.

[4] Information on http://www.leafletjs.com

[5] Information on http://www.openstreetmap.org 